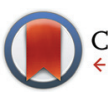

CrossMark \&lick for updates

Cite this: Polym. Chem., 2016, 7, 1129

Received 18th November 2015, Accepted 13th December 2015

DOI: $10.1039 / c 5 p y 01845 e$

www.rsc.org/polymers

\title{
One for all: cobalt-containing polymethacrylates for magnetic ceramics, block copolymerization, unexpected electrochemistry, and stimuli- responsiveness $\uparrow$
}

\author{
C. Rüttiger, $t^{a}$ V. Pfeifer, $t^{a}$ V. Rittscher, ${ }^{a}$ D. Stock, ${ }^{a}$ D. Scheid, ${ }^{a}$ S. Vowinkel, ${ }^{a}$ F. Roth, ${ }^{b}$ \\ H. Didzoleit, ${ }^{c}$ B. Stühn, ${ }^{c}$ J. Elbert, $\S^{a}$ E. Ionescu ${ }^{b}$ and M. Gallei ${ }^{\star a}$
}

\begin{abstract}
Novel cobalt-containing homo- and diblock copolymers with poly(methyl methacrylate) (PMMA) are synthesized by atom transfer radical polymerization (ATRP) of a neutral cobalt-complex methacrylate. An efficient route for a single-step synthesis of the cobalt precursor based on easily-available starting materials followed by esterification with methacrylic acid is presented. The cobalt-methacrylate monomer is furthermore polymerized by thermal, free radical and statistical copolymerization with MMA and investigated with respect to (absolute) molar masses, polymer composition, and thermal properties. ATRP affords block copolymers as evidenced by ${ }^{1} \mathrm{H}$ NMR spectroscopy, size exclusion chromatography (SEC) and differential scanning calorimetry (DSC). The cobalt-containing homopolymers are investigated and tailored with respect to their thermal conversion into magnetic cobalt oxides and elemental cobalt which is evidenced by X-ray diffraction (XRD), Raman spectroscopy, and superconducting quantum interference device (SQUID) magnetometry measurements. The (reversible) electrochemistry of the cobaltcontaining polymethacrylates and block copolymers thereof are thoroughly addressed by cyclic voltammetry (CV) studies. Interestingly, the prepared metalloblock copolymers exhibit redox-responsiveness (both reduction and oxidation) and thus structure formation in the presence of a reduction or oxidation reagent are demonstrated by transmission electron microscopy (TEM).
\end{abstract}

\section{Introduction}

In the recent past, metal-containing polymers attracted enormous attention due to their promising combination of redox, mechanical, semi-conductive, photo-physical, optoelectronic, magnetic and catalytic properties as well as their mechanical robustness. $^{1-13}$ These polymers can either feature a metal center as an integral part of the polymer main chain or the

\footnotetext{
${ }^{a}$ Ernst-Berl-Institut für Technische und Makromolekulare Chemie, Technische Universität Darmstadt, Alarich-Weiss-Str. 4, D-64287 Darmstadt, Germany. E-mail:m.gallei@mc.tu-darmstadt.de

${ }^{b}$ Institute of Condensed Matter Physics, Technische Universität Darmstadt, Hochschulstr. 8, D-64289 Darmstadt, Germany

${ }^{c}$ Materials Science Department, Technische Universität Darmstadt, JovankaBontschits-Str. 2, D-64287 Darmstadt, Germany

$\dagger$ Electronic supplementary information (ESI) available: Additional data on general characterization methods, materials, monomer and polymer syntheses, NMR spectra, molecular weight distributions, Raman spectral indicators, DSC thermograms, SQUID measurement, cyclic voltammetry studies. See DOI: 10.1039/c5py01845e

$\$$ These authors contributed equally.

$\S$ Present address: Department of Chemical Engineering, Massachusetts Institute of Technology, 77 Massachusetts Avenue, Cambridge, MA, 02139, USA.
}

metal-containing moiety is laterally attached to the polymer chain. The vast majority of reports on metal-containing polymers deal with metallocene-based polymers, i.e., metal centres sandwiched between cyclic hydrocarbon moieties, as recent synthetic pathways - mainly focussing on ferrocene monomers - led to stable and well characterized functional materials. In the 1970s Pittman and co-workers succeeded in (co)polymerizing ferrocenyl (meth)acrylates with different monomers by free radical polymerization (FRP) and anionic polymerization, ${ }^{14-17}$ while anionic block copolymerization has been reported more recently. ${ }^{18,19}$ A breakthrough in the synthesis of metallopolymers represents the ring-opening polymerization (ROP) of strained ansa-metallocenophanes found by Manners and coworkers, yielding interesting main-chain metallopolymers. ${ }^{20-22}$ Since Manners' discovery, high-molecular weight polyferrocenylsilanes (PFS) and manifold PFS-based block copolymers with interesting properties were synthesized. Notwithstanding the obstacles in the past, the synthesis of side chain metallopolymers is a field of growing interest. Excellent reviews in that field comprising the synthesis as well as novel applications for ferrocene- and cobaltocene-containing polymers were given by Tang and co-workers. ${ }^{23-25}$ Additionally, ferro- 
cene- and cobalt-containing metallopolymers were found to be feasible as precursors for the preparation of magnetic nanoparticles. ${ }^{26-31}$

Block copolymers consist of two or more polymer segments covalently connected to each other and they have attracted enormous attention in the recent past due to their unique capability of forming fascinating nanostructures in the bulk or by self-organization in solvents. ${ }^{32-38}$ Therefore, applications of block copolymers are manifold ranging, e.g. from fields of nanolithography, drug delivery to separation technologies. Metalloblock copolymers have been used for a variety of interesting applications, which have been reviewed by Manners et al. very recently. ${ }^{39}$ For example, Tang et al. reported on the successful synthesis and self-assembly of triblock copolymers featuring a poly(ferrocenyl methacrylate) block segment for the preparation of ordered iron oxides. ${ }^{40}$

Compared to ferrocene-containing polymers, much less research is carried out in the field of cobalt-containing polymers. In particular the studies on the 18-electron cobaltocenium complex have garnered a great deal of attention. The important difference compared to the isoelectronic ferrocene moiety is the presence of a charge on these complexes and the corresponding metallopolymers which dictates solubility and properties. Excellent reviews within this field of research are given by Tang and co-workers. ${ }^{24,25}$ Very recently, Zhang et al. reported on the formation of anion-responsive cobaltoceniumbased polyelectrolytes with potential applications in the field of healthcare and environmental treatment. ${ }^{41}$ Neutral cobalt(I)-containing metallopolymers consisting of mixed cyclopentadienyl-cobalt-cyclobutadienyl (CpCoCb) complexes were reported by the group of Ragogna. ${ }^{42}$ Recently, a reversible addition-fragmentation transfer (RAFT) polymerization of a $\mathrm{Co}(\mathrm{I})$-acrylate derivative was used for the preparation of low molar mass cobalt-containing block copolymers. ${ }^{43}$

In the present study, the preparation of mixed CpCoCb-containing methacrylate and polymerization (free radical, copolymerization, and atom transfer radical polymerization) and block copolymerization are described. The monomer precursor ( $\eta^{5}$-cyclopentadienyl-4-hydroxybutan-1-on)cobalt $\left(\eta^{4}\right.$-tetraphenylcyclobutadiene) is prepared by a one-step synthesis followed by Steglich esterification. The CpCoCb methacrylate (CpCoCbMA) monomer and the corresponding cobalt-metallopolymer are depicted in Fig. 1.

In addition to the structural and thermal characterization of the synthesized homo- and diblock copolymers involving NMR analysis, size exclusion chromatography (SEC), SEC with multi-angle laser light scattering (SEC-MALLS) detector, differential scanning calorimetry (DSC), and thermal gravimetric analysis (TGA), insights into their electrochemistry and ceramization behavior are presented. The resulting magnetic ceramic is investigated for the first time with respect to its composition and magnetic properties by X-ray diffraction, Raman spectroscopy, and superconducting quantum interference device (SQUID) magnetometry. Inspired by our cyclic voltammetry studies, the cobalt-containing diblock copolymers are investigated regarding their stimulus-responsiveness towards oxi-

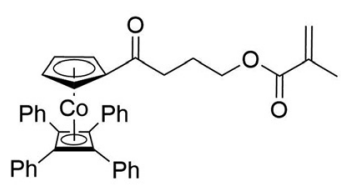

CpCoCbMA

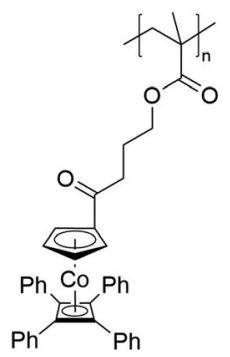

Poly(CpCoCbMA)
Fig. 1 The cobalt-containing methacrylate ( $\mathrm{CpCoCbMA}$, left) and the corresponding poly(CpCoCbMA) (right), which are investigated in this study.

dation and reducing reagents and their unprecedented structure formation as investigated by transmission electron microscopy (TEM).

\section{Results and discussion}

\section{Monomer synthesis}

The synthesis of the cobalt-containing monomer precursor $\left(\eta^{5}\right.$-cyclopentadienyl-4-hydroxybutan-1-on) cobalt $\left(\eta^{4}\right.$-tetraphenylcyclobutadiene) 3 and its esterification with methacrylic acid are shown in Fig. 2. The ring-opening reaction of $\gamma$-butyrolactone 2 with sodium cyclopentadienylide $(\mathrm{NaCp}, 1)$ followed by the reaction with $\mathrm{CoCl}\left(\mathrm{PPh}_{3}\right)_{3}$ and cyclooctadiene (COD) for the preparation of mixed and stable CpCoCOD complexes was reported by Gleiter et al. ${ }^{44}$ and Sigman et al. ${ }^{45}$ Ragogna and coworkers further developed a route for the synthesis of the mixed cyclopentadienyl-cobalt-cyclobutadienyl ( $\mathrm{CpCoCb})$ complex 3 by using a multi-step reaction with the COD-cobalt complex as an intermediate. ${ }^{43}$ Inspired by the synthesis of different mixed CpCoCb complexes by Anderson et al., ${ }^{46}$ we developed a more convenient single-step synthesis for derivative 3 , i.e. ( $\eta^{5}$-cyclopentadienyl-4-hydroxybutan-1-on) cobalt $\left(\eta^{4}\right.$ -

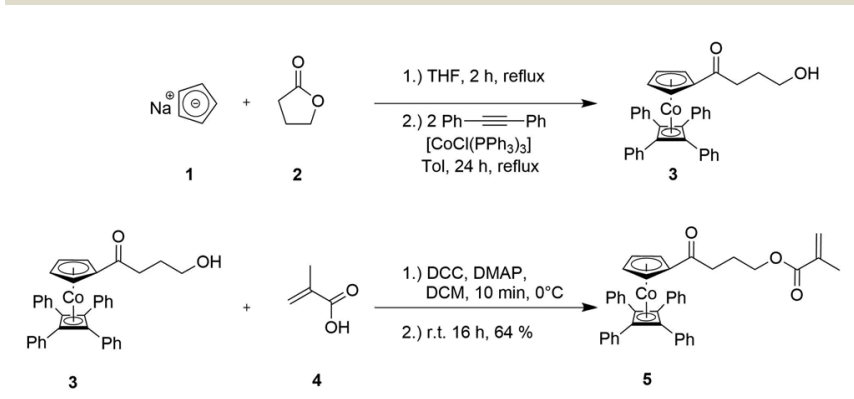

Fig. 2 One-pot synthesis of $\left(\eta^{5}\right.$-cyclopentadienyl-4-hydroxybutan-1on)cobalt $\left(\eta^{4}\right.$-tetraphenyl-cyclobutadiene) $3(\mathrm{CpCoCb})$ by sequential addition of given reagents (top, see the ESI $\dagger$ ). Steglich esterification of $\mathrm{CpCoCb} 3$ with methacrylic acid 4 yielded the cobalt-methacrylate monomer 5 CpCoCbMA. 

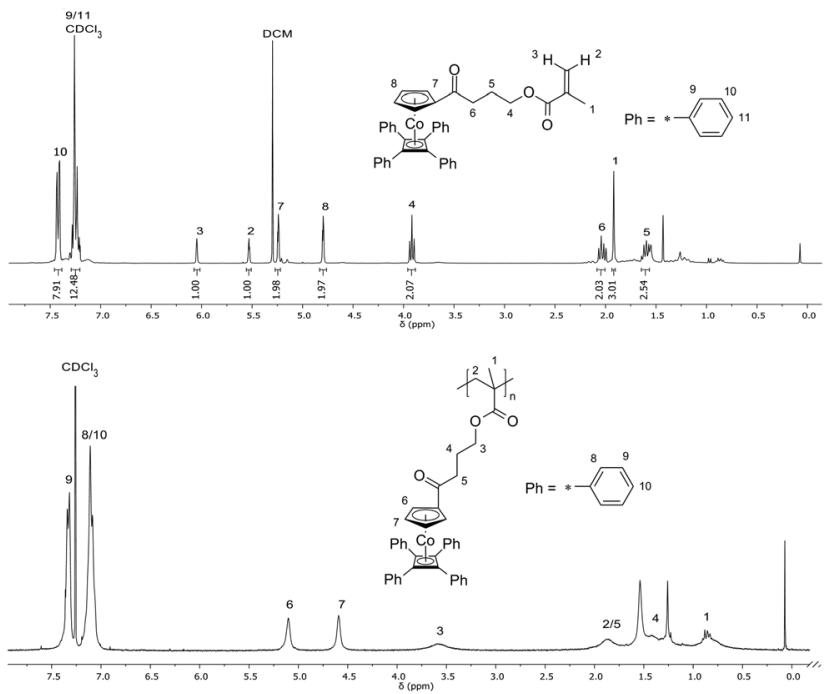

Fig. $3{ }^{1} \mathrm{H}$ NMR spectra and signal assignment of the cobalt-methacrylate monomer 5 ( $\mathrm{CpCoCbMA)} \mathrm{(top)} \mathrm{and} \mathrm{of} \mathrm{a} \mathrm{corresponding} \mathrm{poly}$ ( $\mathrm{CpCoCbMA)} \mathrm{obtained} \mathrm{upon} \mathrm{thermal} \mathrm{polymerization} \mathrm{of} 5$ (bottom).

tetraphenyl-cyclobutadiene), as given in Fig. 2 (top) and as described in detail in the ESI. $\dagger$

The cobalt-containing hydroxyl derivative 3 was used in a Steglich esterification protocol with methacrylic acid (see the ESI $\dagger$ ) in order to obtain the novel cobalt-containing methacrylate 5 (CoCpCbMA) which has not been described in the literature yet. The structure of the obtained monomer $\mathbf{5}$ was supported by ${ }^{1} \mathrm{H}$ NMR spectroscopy (Fig. 3), ${ }^{13} \mathrm{C}$ NMR spectroscopy (Fig. S1 $\dagger$ ), and electrospray ionization mass spectrometry (ESI-MS).

\section{Thermal and free radical polymerization of CPCoCbMA}

Thermal polymerization of the new cobalt-containing methacrylate monomer 5 for the preparation of poly(CpCoCbMA) was performed under vacuum at $100{ }^{\circ} \mathrm{C}$. The obtained orange solid was soluble in common organic solvents, e.g. chloroform and THF. SEC measurements are given in Fig. S2. $\dagger$ As a result, remarkably high molar masses of up to $192 \mathrm{~kg} \mathrm{~mol}^{-1}\left(M_{\mathrm{n}}\right)$ with a polydispersity value, $D$, of 3.99 were obtained for SEC measurements vs. polystyrene (PS) standards. Absolute molar masses were determined for samples obtained by free radical polymerisation using azobisisobutyronitrile (AIBN) as an initiator as described in the ensuing section. An exemplary ${ }^{1} \mathrm{H}$ NMR spectrum of the polymer sample obtained by thermally induced polymerization of monomer $\mathbf{5}$ was directly compared to the corresponding ${ }^{1} \mathrm{H}$ NMR spectrum of monomer 5 in Fig. 3 (bottom). The significant signal broadening and the absence of unsaturated methacrylate proton signals proved the presence of poly(CpCoCbMA).

Free radical polymerization and copolymerization of monomer 5 with methyl methacrylate were performed by initiation with AIBN in toluene at $60{ }^{\circ} \mathrm{C}$ for $24 \mathrm{~h}$ (see the ESI $\dagger$ ). The poly(CpCoCbMA) homopolymers were poured into metha- nol for precipitation and the resulting yellow powder was analysed by SEC measurements, NMR spectroscopy, and differential scanning calorimetry. While the NMR results indicate a similar molecular structure to that of poly(CpCoCbMA) which was obtained by thermal polymerization, only a rather low apparent molar mass, i.e. $8900 \mathrm{~g} \mathrm{~mol}^{-1}\left(M_{\mathrm{n}}\right)$, was determined by using SEC vs. PS calibration for poly-(CpCoCbMA) (Fig. S3†). Absolute molar masses which were determined by SEC with multi-angle laser light scattering (SEC-MALLS) detection for these polymers will be described in the ensuing section. The novel poly(CpCoCbMA) was investigated with respect to its thermal properties by DSC (Fig. S4†). It can be concluded from Fig. $\mathrm{S} 4 \uparrow$ that only one glass transition temperature, $T_{\mathrm{g}}$, was observed at $T_{\mathrm{g}}=137^{\circ} \mathrm{C}$, which is higher compared to that of similar cobalt-containing poly(acrylates) reported in the literature, which were obtained by RAFT polymerization $\left(T_{\mathrm{g}}=85^{\circ} \mathrm{C}\right) .{ }^{43}$ Additionally, radical copolymerization with methyl methacrylate (MMA) was performed as described in the ESI. $\dagger$ The results on SEC, DSC, and ${ }^{1} \mathrm{H}$ NMR spectroscopy measurements are given in Fig. S5-S7, $\dagger$ respectively. Based on these results, it can be concluded that also the copolymerization of CpCoCbMA 5 with MMA was successful, as supported by a monomodal molecular weight distribution as well as the presence of a $T_{\mathrm{g}}$ value of $120^{\circ} \mathrm{C}$, hence between the $T_{\mathrm{g}}$ of pure poly(CpCoCbMA) $\left(137{ }^{\circ} \mathrm{C}\right)$ and pure PMMA obtained by free radical polymerization $\left(105^{\circ} \mathrm{C}\right) .{ }^{47}$ Moreover, based on the corresponding ${ }^{1} \mathrm{H}$ NMR spectrum (Fig. S7†), a PMMA content of $56 \mathrm{~mol} \%$ and $44 \mathrm{~mol} \%$ for poly(CpCoCbMA) could be determined proving the success of radical copolymerization of MMA and the cobalt-containing monomer CpCoCbMA 5.

\section{Atom transfer radical polymerization of CpCoCbMA and block copolymer formation}

Atom transfer radical polymerization (ATRP) of CpCoCbMA 5 was carried out with 2-bromoisobutyric tert-butyl ester ( $t$ Bbib) as an ATRP initiator and [Cu$\left.{ }^{\mathrm{I}}(\mathrm{PMDETA}) \mathrm{Br}\right]$ as a catalyst in anisole at $80{ }^{\circ} \mathrm{C}$ (see the ESI $\dagger$ ). The synthesis is depicted in Fig. 4 (top). As a result, narrowly distributed molecular weight distributions can be obtained for poly-(CpCoCbMA) as determined by SEC measurements (Fig. S8†), thus proving the feasibility of the applied ATRP protocols. Exemplarily, an apparent molar mass of $5800 \mathrm{~g} \mathrm{~mol}^{-1}$ was obtained by using SEC vs. PS standards.

Before the block copolymerization of MMA and CpCoCbMA 5 was investigated, we studied the absolute molar masses of poly(CpCoCbMA), which were obtained by free radical polymerization and ATRP. The results are compiled in Table 1 for direct comparison. It turned out that the absolute molar masses of poly(CpCoCbMA) significantly differ from the values obtained by SEC vs. PS standards. In other words, poly(CpCoCbMA) chains feature considerably smaller hydrodynamic volumes compared to e.g. PS chains of identical contour length. This is maybe due to the fact that poly(CpCoCbMA) chains are rather weakly solvated in common solvents for SEC analysis like THF. This behaviour was also found for other 


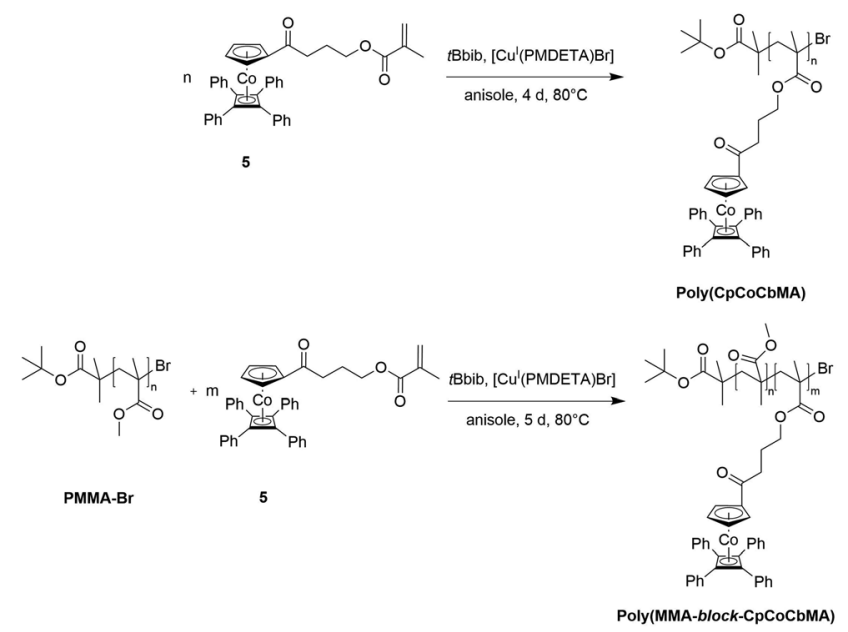

Fig. 4 Atom transfer radical polymerization (ATRP) of CpCoCbMA 5 (top) and block copolymerization starting with a PMMA-Br macroinitiator (bottom) for the preparation of PMMA-block-P(CpCoCbMA).

Table 1 Comparison of relative and absolute molar masses for poly ( $\mathrm{CpCoCbMA)}$ as obtained by free radical polymerization and ATRP

\begin{tabular}{lllll}
\hline & $\begin{array}{l}M_{\mathrm{n}}{ }^{a} \\
\left(\mathrm{~g} \mathrm{~mol}^{-1}\right)\end{array}$ & $\begin{array}{c}M_{\mathrm{w}}{ }^{a} \\
\left(\mathrm{~g} \mathrm{~mol}^{-1}\right)\end{array}$ & $\begin{array}{l}M_{\mathrm{w}}{ }^{b} \\
\left(\mathrm{~g} \mathrm{~mol}^{-1}\right)\end{array}$ & $D^{a}$ \\
\hline Poly(CpCoCbMA $)^{c}$ & 8900 & 18600 & 56000 & 2.09 \\
${\text { Poly(CPCoCbMA })^{d}}^{c}$ & 4600 & 5800 & 12800 & 1.26
\end{tabular}

${ }^{a}$ Molar masses and $D$ determined by using SEC measurements with PS calibration. ${ }^{b}$ Molar masses determined by using SEC-MALLS measurements. Refractive index increment was determined to $\mathrm{d} n / \mathrm{d} c=$ 0.169. ${ }^{c}$ Polymer obtained by free radical polymerization with AIBN as an initiator. ${ }^{d}$ Polymer obtained by ATRP.

metallopolymers with sterically demanding pendant groups, e.g. polyvinylferrocene ${ }^{48}$ or poly(2-(methacryloyloxy)ethyl ferrocenecarboxylate) (PFcMA). ${ }^{28}$

For the preparation of block copolymers, PMMA-Br macroinitiators were prepared via ATRP using 2-bromoisobutyric tertbutyl ester $(t \mathrm{Bbib})$ as the initiator and $\left[\mathrm{Cu}^{\mathrm{I}}(\mathrm{TMEDA})_{2} \mathrm{Br}\right]$ as the catalyst system in anisole at $88{ }^{\circ} \mathrm{C}$ (Fig. 4, bottom, for details refer to the ESI $\dagger$ ). The PMMA-Br macroinitiators were precipitated in $n$-hexane, filtered and dried in vacuo. PMMA-Br was used for the initiation of monomer CpCoCbMA 5 in anisole in the presence of $\left[\mathrm{Cu}^{\mathrm{I}}(\mathrm{PMDETA})_{2} \mathrm{Br}\right]$ at $80{ }^{\circ} \mathrm{C}$. After $5 \mathrm{~d}$ of reaction time, the corresponding polymers were again precipitated, filtered and dried in vacuo. Exemplary results on block copolymerization obtained by SEC measurements for the PMMA-Br macro-initiators and the final cobalt-containing block copolymers are given in Fig. 5 (PMMA-Br, and block 1) and Fig. S9† (block 2).

The compositions of the final PMMA- $b$-PCpCoCbMA block copolymers were determined by using ${ }^{1} \mathrm{H}$ NMR spectroscopy (Fig. S10 and S11†). All results on the corresponding block copolymers are compiled in Table 2. A first evidence that these

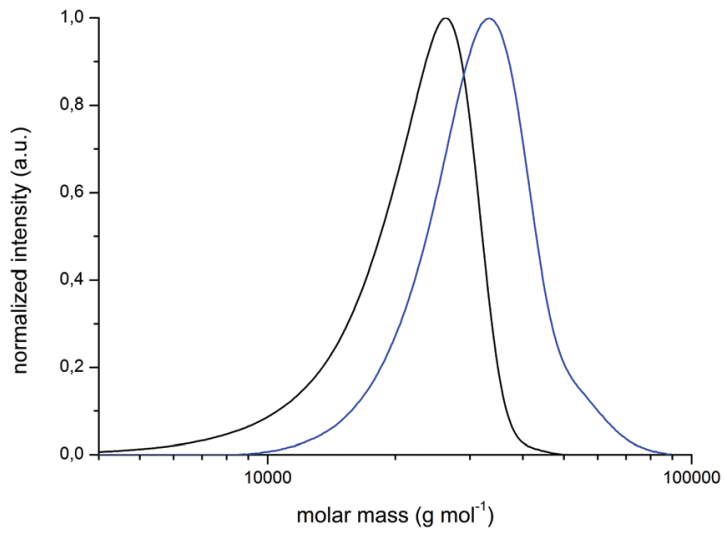

Fig. 5 Molecular weight distribution of poly(MMA-block-CpCoCbMA) in THF (blue curve) obtained by sequential ATRP of the PMMA-Br macro-initiator (black curve) with CpCoCbMA 5. The obtained values were calibrated against PS as standards.

Table 2 Molar masses obtained by SEC measurements vs. PS standards for ATRP block copolymerization of MMA and CpCoCbMA

\begin{tabular}{lrllll}
\hline & $\begin{array}{l}M_{\mathrm{n}}{ }^{a} \\
\left(\mathrm{~g} \mathrm{~mol}^{-1}\right)\end{array}$ & $\begin{array}{l}M_{\mathrm{w}}{ }^{a} \\
\left(\mathrm{~g} \mathrm{~mol}{ }^{-1}\right)\end{array}$ & $D$ & $\begin{array}{l}\text { Comp. } \\
(\mathrm{wt} \%)\end{array}$ & $T_{g}\left({ }^{\circ} \mathrm{C}\right)$ \\
\hline PMMA-Br 1 & 19600 & 22600 & 1.15 & $100 / 0$ & $116^{\circ} \mathrm{C}$ \\
PMMA-Br 2 & 9500 & 12100 & 1.27 & $100 / 0$ & $116^{\circ} \mathrm{C}$ \\
Block 1 & 28800 & 30100 & 1.05 & $73 / 27$ & $116^{\circ} \mathrm{C} / 134^{\circ} \mathrm{C}$ \\
Block 2 & 13000 & 15000 & 1.15 & $57 / 43$ & $116^{\circ} \mathrm{C} / 133^{\circ} \mathrm{C}$
\end{tabular}

${ }^{a}$ Molar masses determined by using SEC measurements vs. PS calibration. ${ }^{b}$ Comp.: compositions were determined by using ${ }^{1} \mathrm{H}$ NMR spectroscopy; values are given as wt $\%$ PMMA to wt $\%$ P(CpCoCbMA).

block copolymers are capable of microphase separation is due to the presence of two glass transition temperatures, i.e. $116{ }^{\circ} \mathrm{C}$ which can be assigned to the PMMA block segment and $134{ }^{\circ} \mathrm{C}$ which corresponds to poly(CpCoCbMA). The corresponding DSC differential-thermograms can be found in Fig. S12 and S13, $\dagger$ respectively.

In summary, ATRP is a feasible method for the preparation of poly(CpCoCbMA)-containing block copolymers with PMMA as the first block segment. Moreover, excellent control over block copolymer composition with low values on polydispersity ( $D$ between 1.05 and 1.15) was achieved. Before the stimuli-responsiveness of the cobalt-containing block copolymers is investigated, ceramization of the corresponding cobaltcontaining homopolymers and their interesting electrochemistry will be addressed in the ensuing two sections.

\section{Thermal conversion of poly(CpCoCbMA) into cobalt- containing magnetic ceramics}

As already mentioned in the Introduction, metallopolymers are excellent candidates for the preparation of advanced functional ceramics after thermal treatment. Therefore, the cobaltcontaining homopolymers were investigated with respect to their thermal behaviour under different atmospheres, i.e. 


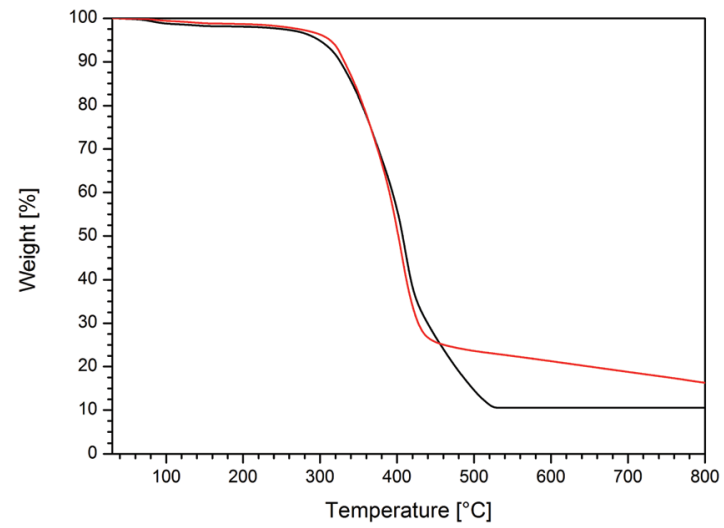

Fig. 6 TGA curves of poly( $\mathrm{CpCoCbMA)} \mathrm{performed} \mathrm{with} \mathrm{a} \mathrm{heating} \mathrm{rate}$ of $10 \mathrm{~K} \mathrm{~min}^{-1}$ starting from room temperature to $800^{\circ} \mathrm{C}$ under oxygen (black curve) and nitrogen (red curve) atmospheres.

nitrogen and oxygen, by using thermal gravimetric analysis (TGA). Exemplary diagrams for the thermal treatment of poly(CpCoCbMA) either under a nitrogen or oxygen atmosphere with a heating rate of $10 \mathrm{~K} \mathrm{~min}^{-1}$ in the range from room temperature up to $800{ }^{\circ} \mathrm{C}$ are shown in Fig. 6. A similar thermal behaviour of poly(CpCoCbMA) under both atmospheres can be concluded at moderate temperatures (i.e., up to ca. $430{ }^{\circ} \mathrm{C}$ ), with a significant weight loss starting at $320{ }^{\circ} \mathrm{C}$. However, the thermal treatment under an oxygen atmosphere revealed a more pronounced weight loss up to a temperature of $520^{\circ} \mathrm{C}$ (black curve, Fig. 6), as compared to the ceramization process performed under a nitrogen atmosphere (red curve, Fig. 6). Interestingly, the weight loss of poly(CpCoCbMA) occurred slower compared to the sample treated under an oxygen atmosphere. Moreover, the sample treated under a nitrogen atmosphere was shown to further decompose on increasing the soaking time at $800{ }^{\circ} \mathrm{C}$. Thus, 30 additional minutes of annealing at $800{ }^{\circ} \mathrm{C}$ resulted in an increase of the mass loss from 83.7 to $89.9 \mathrm{wt} \%$. Noteworthily, the sample treated under a nitrogen atmosphere showed a magnetic response with a commercially available magnet, while this was not the case for the oxygen-treated sample of poly(CpCoCbMA) after heating to $800{ }^{\circ} \mathrm{C}$. As the magnetic sample was of special interest, the nitrogen-treated sample was additionally investigated by using X-ray diffraction (XRD), Raman spectroscopy, and SQUID (superconducting quantum interference device) magnetometry, which are described in the following section.

The X-ray diffraction (XRD) pattern of the sample obtained from poly(CpCoCbMA) heat-treated under a nitrogen atmosphere at $800{ }^{\circ} \mathrm{C}$ is given in Fig. 7. The reflections can be assigned to three different phases comprising: $43.4 \% \mathrm{CoO}$, 46.8\% $\mathrm{Co}_{3} \mathrm{O}_{4}$ and 9.9\% Co. Only strong reflections are labelled in Fig. 7. The full profile is analysed using the Rietveld algorithm. The $\mathrm{CoO}$ structure shows a cubic structure with the $F m \overline{3} m$ space group ${ }^{49}$ and a unit cell length of (4.3 $⿱$ A). Interestingly, the $\mathrm{Co}_{3} \mathrm{O}_{4}$ contribution could be matched by a cubic system in the $F d \overline{3} m$ space group with a unit cell length of 8.1 A. The elemental cobalt phase was assigned to a cubic system in the $F m \overline{3} m$ space group with a side length of $3.5 \AA$ A. Compared to these findings, the same sample heat-treated under an oxygen atmosphere yielded pure $\mathrm{Co}_{3} \mathrm{O}_{4}$ as could be concluded from the XRD pattern (Fig. S14 $\dagger$ ).

In order to gain more insights into the composition of the magnetic material, Raman spectroscopy was performed additionally. The corresponding Raman spectrum of poly(CpCoCbMA) which was thermally treated at $800^{\circ} \mathrm{C}$ under a nitrogen atmosphere is given in Fig. 8 and indicates the presence of the spinel-type $\mathrm{Co}_{3} \mathrm{O}_{4}$ phase. Considering the spinel

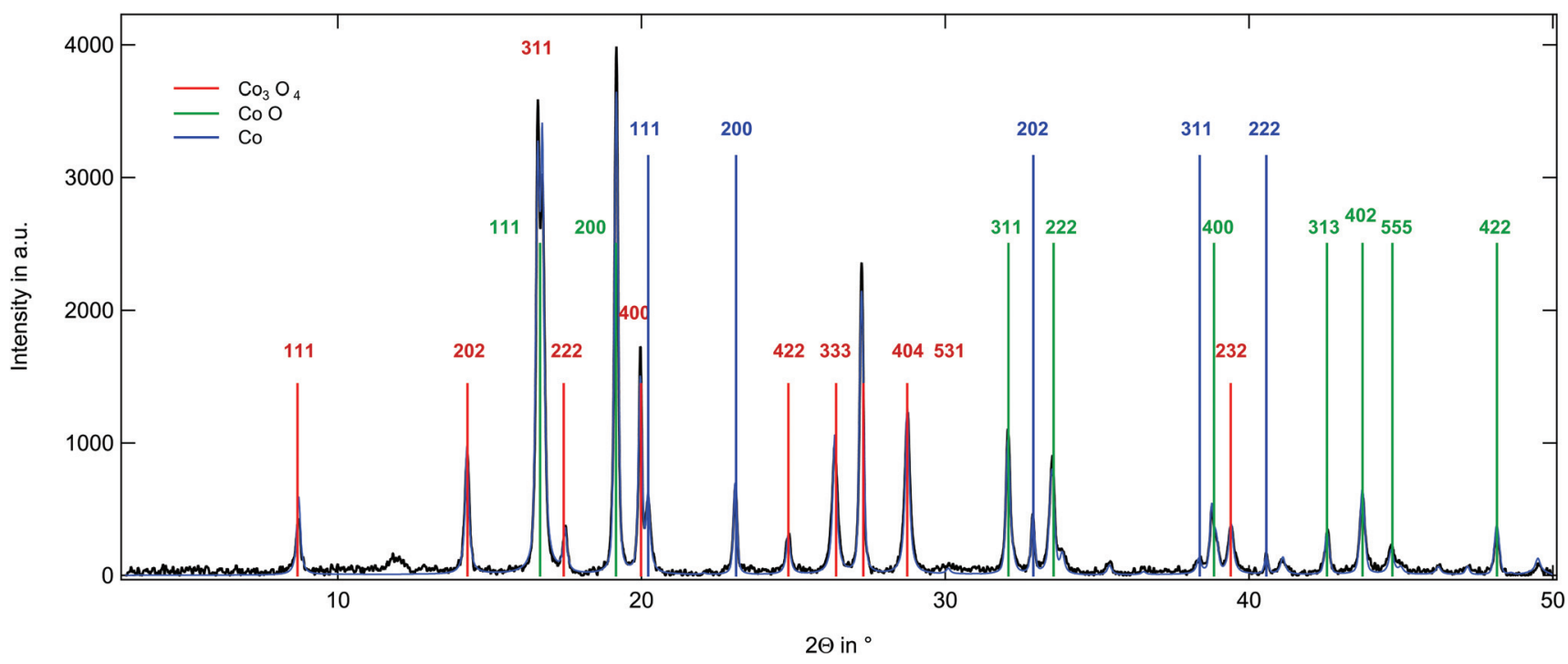

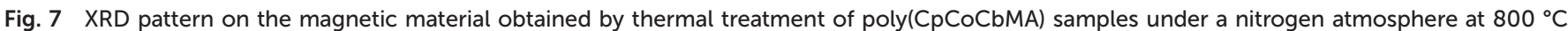
(see the text). 


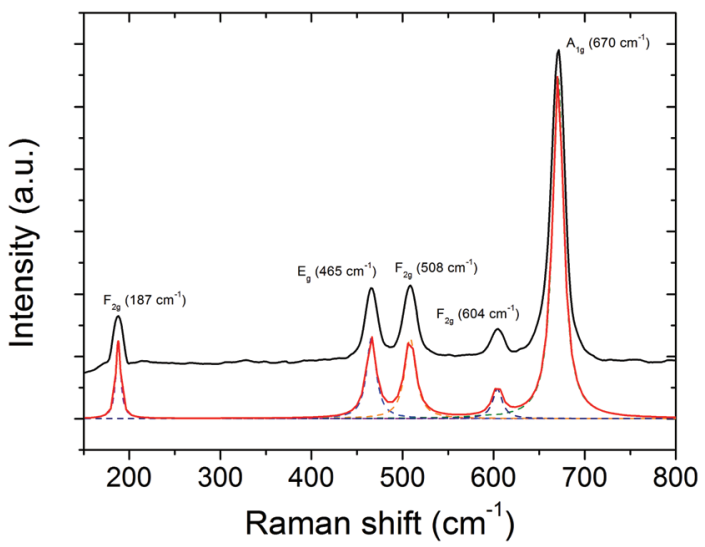

Fig. 8 Raman spectrum of the sample prepared upon pyrolysis of poly $(\mathrm{CpCoCbMA})$ at $800{ }^{\circ} \mathrm{C}$ under a nitrogen atmosphere showing the five Raman-active optical-phonon modes of spinel-type $\mathrm{CO}_{3} \mathrm{O}_{4}$.

structure of $\mathrm{Co}_{3} \mathrm{O}_{4}$ (space group $O_{h}{ }^{7}$ ), five vibrational modes among the total of 16 zone-center optical-phonon modes are Raman active, and were assigned to the absorption bands at $187\left(\mathrm{~F}_{2 \mathrm{~g}}\right), 465\left(\mathrm{E}_{2 \mathrm{~g}}\right), 508\left(\mathrm{~F}_{2 \mathrm{~g}}\right), 604\left(\mathrm{~F}_{2 \mathrm{~g}}\right)$ and $670\left(\mathrm{~A}_{1 \mathrm{~g}}\right) \mathrm{cm}^{-1}$ (Fig. 8). ${ }^{50}$ Interestingly, Co(II) oxide was not detected unambiguously by Raman spectroscopy despite its presence was demonstrated by XRD, as the three corresponding Raman active modes thereof (at 475,600 and $680 \mathrm{~cm}^{-1}$ ) ${ }^{51-53}$ were weak in intensity and probably overlap with the absorption bands of the spinel-type $\mathrm{Co}_{3} \mathrm{O}_{4}$. Similar findings related to difficulties of detecting $\mathrm{CoO}$ by Raman spectroscopy in the presence of $\mathrm{Co}_{3} \mathrm{O}_{4}$ were repeatedly reported in the literature. ${ }^{51,54-57}$ Furthermore, the sensitivity of $\mathrm{CoO}$ towards the laser beam (which might induce its conversion into $\mathrm{Co}_{3} \mathrm{O}_{4}$ ) can be considered as an additional reason for the ambiguous detection thereof by Raman spectroscopy. ${ }^{54,55}$

These findings based on XRD and Raman spectroscopy results clearly revealed the feasibility of the herein investigated cobalt-containing metallopolymers as excellent preceramic polymer precursors for the synthesis of Co-containing magnetic ceramics. In order to investigate the magnetic properties, SQUID measurement on the material was carried out. The corresponding SQUID measurement is shown in Fig. S15. $\dagger$ As a conclusion on these data, the ceramic material indicates a ferromagnetic behaviour with an average remanence magnetisation of $M_{\mathrm{r}}=722 \mathrm{emu} \mathrm{g}^{-1}$. The corresponding rather low coercivity field revealed a strength of $H_{\mathrm{c}}=30$ Oe for the corresponding upper and the lower curve. This value is in agreement with experiments on cobalt films. ${ }^{58}$ Due to instrumental limitations, a saturation magnetisation could not be determined. In summary, the herein followed convenient route paves the way for the preparation of novel polymer-derived magnetic ceramics. Moreover, $\mathrm{Co}_{3} \mathrm{O}_{4}$-based materials are promising candidates as anode materials for lithium secondary batteries due to their high electrochemical capacity and excellent recharge rates. ${ }^{59}$ Soft polymer templating will enable the preparation of tailored architectures, adjustable material composition, and the application on doping protocols for the preparation of functional ceramics which is currently under investigation.

\section{Electrochemistry of poly(CpCoCbMA)}

Besides the ability to form ceramics, another interesting property of metallopolymers is their fascinating redox-responsiveness due to the presence of metal centers capable of featuring several oxidation levels. While the redox chemistry of ferrocene- and cobaltocene-containing polymers has been studied leading to a plethora of fascinating applications, ${ }^{60-64}$ reports on the redox properties of neutral CpCoCb-containing polymers have not been reported yet. Studies on the electrochemistry of single $\mathrm{CpCoCb}$ complexes showed that these compounds were capable of both reduction and oxidation. ${ }^{65,66}$ For the $\mathrm{CpCoCb}$ without substituents at the cyclopentadienyl rings, a reversible oxidation at $+0.91 \mathrm{~V}$ to the corresponding monocation, followed by an irreversible oxidation at +1.55 was reported by Koelle. ${ }^{66}$ Brotin and co-workers reported that this complex can be reduced at $-2.24 \mathrm{~V}$ yielding an anion with the charge mainly localised on the cyclobutadienyl ligand. ${ }^{65}$ This unprecedented possibility to switch neutral $\mathrm{CpCoCb}$ complexes in two ways by electrochemistry encouraged us to further study our novel cobalt polymers. Therefore, cyclic voltammetry (CV) studies of poly(CpCoCbMA) in dichloromethane were carried out. CV curves showed a partially reversible oxidation at $+1.24 \mathrm{~V}$ (Fig. 9, bottom) with a ratio of the peak currents $i_{\mathrm{p}}^{\text {red }} / i_{\mathrm{p}}^{\text {ox }}=0.4$. The reduced reversibility after $10 \mathrm{CV}$ cycles can be attributed to the low solubility of the positively charged polymer in dichloromethane leading to a precipitation of the oxidized polymer. In fact, we could observe a reversible oxidation for the monomer in dichloromethane at $+1.05 \mathrm{~V}$ (Fig. 9, top), indicating an electrochemical reversibility of the $\mathrm{CpCoCb} / \mathrm{CpCoCb}^{+}$redox couple in poly(CpCoCbMA). The shift of this redox couple towards higher oxidation potentials of CpCoCbMA compared to the unsubstituted $\mathrm{CpCoCb}$ (Fig. S16 $\dagger$ ) can be attributed to the electron withdrawing nature of the butanoyl substituent on the cyclopentadienyl ligand. Due to the presence of signals during CV measurements of the $\mathrm{CpCoCb}$ complex for reductive potentials in dichloromethane, we studied the response towards reduction for poly(CpCoCbMA) in $\mathrm{CH}_{2} \mathrm{Cl}_{2}$ (Fig. $\mathrm{S} 16, \uparrow$ bottom). A small peak at $-1.45 \mathrm{~V}$ indicated that the reduction of the polymer to the anionic species is possible. For unsubstituted $\mathrm{CpCoCb}$, we found a reversible reduction at $-0.90 \mathrm{~V}$ (Fig. S16 $\dagger$ ). In contrast to the $\mathrm{CpCoCb} / \mathrm{CpCoCb}^{+}$redox couple, no influence of the substituent on the $\mathrm{Cp}$ ligand on the potential of the $\mathrm{CpCoCb}^{-} /$ $\mathrm{CpCoCb}$ redox couple could be observed, which is attributed to the localisation of the negative charge on the cyclobutadienyl ligand. ${ }^{65}$

\section{Structure formation of cobalt-containing block copolymers after reaction with redox-reagents in solution}

To show the redox-responsive character of the novel cobalt-containing block copolymers, samples of PMMA- $b$-PCpCoCbMA (block 1) were dissolved in a small amount of $\mathrm{CHCl}_{3}$ and 

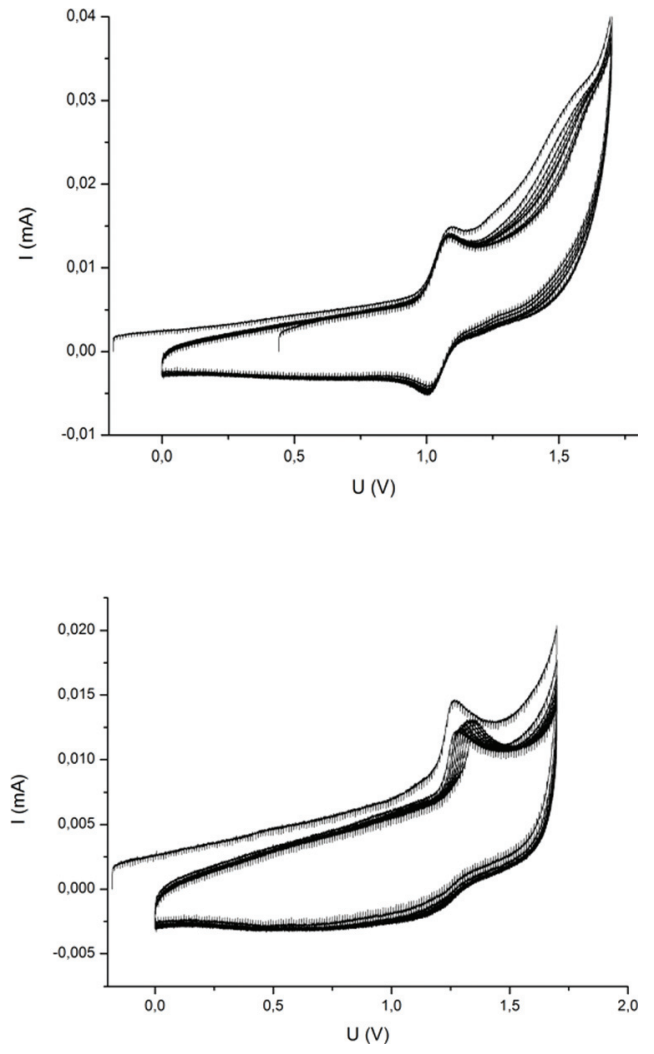

Fig. 9 Repeated cyclic voltammetry curves $(10$ cycles $)$ of the CpCoCbMA monomer (top) and poly(CpCoCbMA) (bottom) in $\mathrm{CH}_{2} \mathrm{Cl}_{2}$ with TBAHFP (0.1 M) as an electrolyte. Scan rate was $20 \mathrm{mV} \mathrm{s}^{-1}$.

treated with either a reducing or an oxidation reagent (see the ESI $\dagger$ ). Inspired by the results on redox waves and potentials obtained by the $\mathrm{CV}$ measurements as described above, $\mathrm{NOBF}_{4}$ was used as an oxidation reagent and decamethycobaltocene $\left(\mathrm{Cp}_{2}{ }^{*} \mathrm{Co}\right)$ as a reducing agent for redox-controlled micellation of the cobalt-containing block copolymers. As a first hint that chemical reactions took place, the block copolymer solution changed in colour after treatment with a reducing or oxidation reagent, respectively. To gain insights into the formation of micellar aggregates, TEM images of the micellar solutions were recorded. For this purpose, the samples were drop-cast on a carbon-coated copper grid followed by drying under ambient conditions or argon flow prior to the TEM measurements. The corresponding results on TEM measurements are given in Fig. 10. Due to the presence of metallopolymers featuring sufficient electron contrast, there was no need for staining of the samples. In the case of treating the block copolymer solution in $\mathrm{CHCl}_{3}$ with $\mathrm{Cp}_{2}{ }^{*} \mathrm{Co}$, worm-like micellar structures were observed. Spherical and cylindrical micelles revealed diameters of approximately $20-30 \mathrm{~nm}$ as determined by TEM (Fig. 10, top). Noteworthily, treatment of the cobalt-containing block copolymers with $\mathrm{NOBF}_{4}$ in order to oxidize the cobalt-containing segment yielded spherical micellar structures (with $<10 \mathrm{~nm}$ of average diameter) as shown by TEM measurements (Fig. 10, bottom). Compared to these findings, for block copolymer

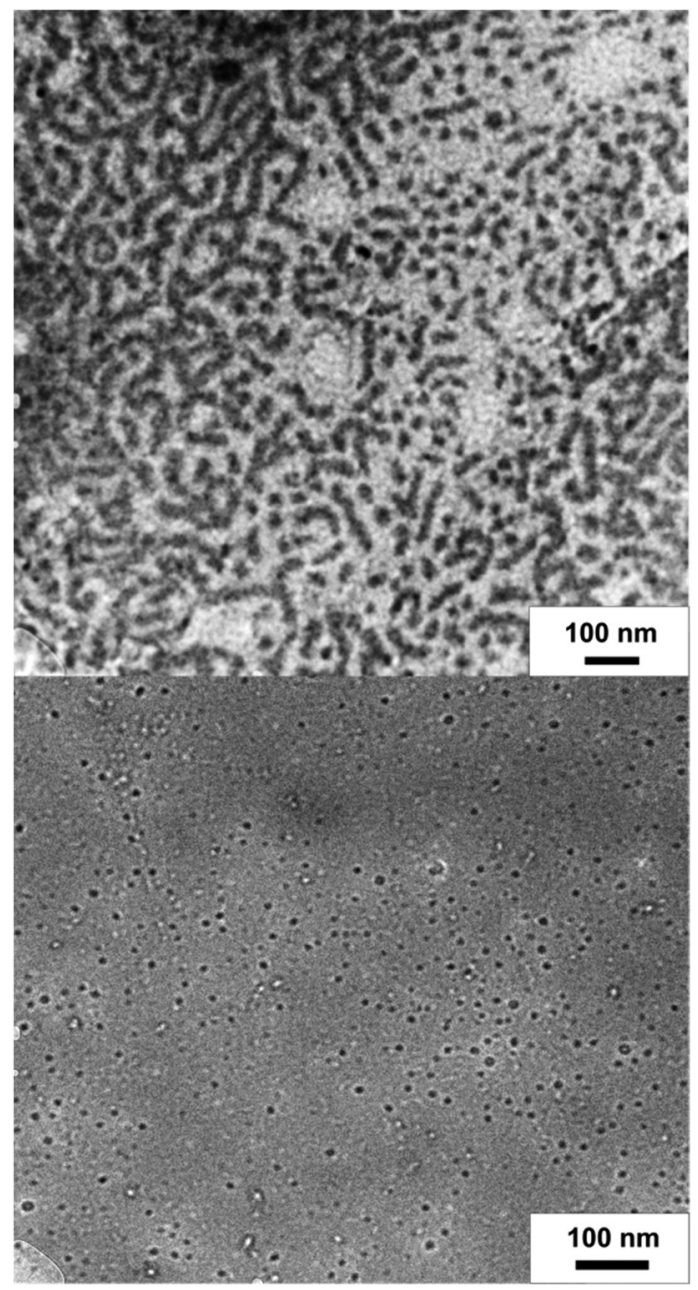

Fig. 10 TEM images of PMMA-block-PCpCoCbMA micelles formed in chloroform after treatment with decamethylcobaltocene $\left(\mathrm{Cp}_{2}{ }^{*} \mathrm{Co}\right)$ (top) or treatment with $\mathrm{NOBF}_{4}$ (bottom). The micelles were deposited on a carbon-coated copper grid followed by drying at ambient temperature. Scale bars correspond to $100 \mathrm{~nm}$.

solutions without the presence of reducing or oxidation reagents, no structure formation could be observed during TEM measurements which point towards well diluted block copolymers in $\mathrm{CHCl}_{3}$. Due to the unpredictable influence of the significant amount of salts present after reduction/oxidation reactions, we did not check for reversible micelle formation.

From the TEM images it can be concluded that worm-like micelles (reduction) or remarkably uniform spherical micelles were obtained similar to previously reported ferrocene-containing block copolymers. ${ }^{67-70}$ It is worth mentioning that the herein investigated novel cobalt-containing block copolymers comprise a feasible platform for switching the same metallopolymer by two different chemical pathways by redox chemistry. This fascinating behavior of these cobalt-containing block copolymers is due to their capability of switching the cobalt moiety either by a reductive route or oxidative route. 


\section{Conclusions}

In the current study, an efficient way for the preparation of a cobalt-containing methacrylate, i.e. CpCoCbMA, has been presented. The successful thermal, free radical and copolymerization (with MMA), and atom transfer radical polymerization (ATRP) has been proved by SEC, SEC-MALLS, NMR spectroscopy, and DSC measurements. The corresponding cobalt-containing homopolymers could be thermally treated under a nitrogen atmosphere to yield magnetic cobalt oxide as evidenced by XRD, Raman spectroscopy, and SQUID measurements. Efficient ATRP protocols for the preparation of poly (CpCoCbMA)-containing block copolymers with PMMA as a block partner were presented. It has been shown by cyclic voltammetry $(\mathrm{CV})$ that the corresponding metallopolymers underwent reversible reduction and oxidation reactions. Finally, the redox-responsiveness of the diblock copolymers was evidenced by treatment with chemical reducing and oxidation reagents leading to interesting structure formation as observed by TEM measurements. The investigated cobalt-containing block copolymers can therefore be used as multi-redox-responsive materials. Our findings will pave the way to an addressable and highly functional platform with unprecedented and potential applications for selective release, nanolithography and for the formation of magnetic devices based on soft polymer templates.

\section{Author contribution}

MG, CR, and JE conceived the idea, CR and VP synthesized the polymers and performed measurements on polymer analytics, VR and JE investigated the electrochemistry, CR, DSch and DSt investigated micellation by TEM, SV was responsible for ceramization and TGA, FR and EI investigated the cobalt oxides by Raman spectroscopy, HD and BS were responsible for SQUID and XRD analysis and interpretation. The manuscript was written through contributions of all authors. All authors have given approval to the final version of the manuscript.

\section{Acknowledgements}

The authors owe Prof. Alan Hatton from the Massachusetts Institute of Technology a debt of gratitude for kind discussion and help on the cyclic voltammetry results. The authors thank Jean-Christoph Jaud (group of Prof. W. Donner) from the Materials Science Department Darmstadt for performing the XRD measurement. The authors cordially thank Prof. M. Biesalski and his group (Macromolecular Chemistry and Paper Chemistry Department, TU Darmstadt) for analytical support. M. G. acknowledges the "Fonds der Chemischen Industrie" and the Max-Buchner Foundation (Dechema). J. E. would like to thank the German Academic Exchange Service (DAAD) for a Postdoctoral fellowship.

\section{Notes and references}

1 A. S. Abd-El-Aziz and I. Manners, Frontiers in Transition Metal-Containing Polymers, Wiley-Interscience, Hoboken, NJ, 1997.

2 D. Wöhrle and A. D. Pomogailo, Metal Complexes and Metals in Macromolecules: Synthesis, Structure and Properties, Wiley-VCH, Weinheim, Germany, 2003.

3 C. E. Carraher, A. S. Abd-El-Aziz, C. Pittman, J. Sheats and M. Zeldin, A Half Century of Metal and Metalloid Containing Polymers, Wiley, New York, 2003.

4 I. Manners, Synthetic Metal-Containing Polymers, VCH, Weinheim, Germany, 2004.

5 G. R. Whittell and I. Manners, Adv. Mater., 2007, 19, 34393468.

6 G. R. Whittell, M. D. Hager, U. S. Schubert and I. Manners, Nat. Mater., 2011, 10, 176-188.

7 R. H. Staff, M. Gallei, M. Mazurowski, M. Rehahn, R. Berger, K. Landfester and D. Crespy, ACS Nano, 2012, 6, 9042-9049.

8 A. S. Abd-El-Aziz and E. A. Strohm, Polymer, 2012, 53, 48794921.

9 J. Elbert, M. Gallei, C. Rüttiger, A. Brunsen, H. Didzoleit, B. Stühn and M. Rehahn, Organometallics, 2013, 32, 58735878.

10 M. Gallei, Macromol. Chem. Phys., 2014, 215, 699-704.

11 A. S. Abd-El-Aziz, C. Agatemor and N. Etkin, Macromol. Rapid Commun., 2014, 35, 513-559.

12 J. Elbert, F. Krohm, C. Rüttiger, S. Kienle, H. Didzoleit, B. N. Balzer, T. Hugel, B. Stühn, M. Gallei and A. Brunsen, Adv. Funct. Mater., 2014, 24, 1591-1601.

13 A. D. Russell, R. A. Musgrave, L. K. Stoll, P. Choi, H. Qiu and I. Manners, J. Organomet. Chem., 2015, 784, 24-30.

14 C. U. Pittman, J. C. Lai and D. P. Vanderpool, Macromolecules, 1970, 3, 105.

15 C. U. Pittman and A. Hirao, J. Polym. Sci., Polym. Chem. Ed., 1977, 15, 1677.

16 C. U. Pittman and A. Hirao, J. Polym. Sci., Polym. Chem. Ed., 1978, 16, 1197.

17 C. U. Pittman and C. C. Lin, J. Polym. Sci., Polym. Chem. Ed., 1979, 17, 271.

18 M. Gallei, B. V. K. J. Schmidt, R. Klein and M. Rehahn, Macromol. Rapid Commun., 2009, 30, 1463-1469.

19 M. Gallei, S. Tockner, R. Klein and M. Rehahn, Macromol. Rapid Commun., 2010, 31, 889-896.

20 D. A. Foucher, B.-Z. Tang and I. Manners, J. Am. Chem. Soc., 1992, 114, 6246-6248.

21 I. Manners, Can. J. Chem., 1998, 76, 371-381.

22 V. Bellas and M. Rehahn, Angew. Chem., Int. Ed., 2007, 46, 5082-5104.

23 C. G. Hardy, L. Ren, T. C. Tamboue and C. Tang, J. Polym. Sci., Part A: Polym. Chem., 2011, 49, 1409-1420.

24 C. G. Hardy, L. Ren, J. Zhang and C. Tang, Israel J. Chem., 2012, 52, 230.

25 C. G. Hardy, J. Zhang, Y. Yan, L. Ren and C. Tang, Prog. Polym. Sci., 2014, 39, 1742-1796. 
26 K. Temple, K. Kulbaba, K. N. Power-Billard, I. Manners, K. A. Leach, T. Xu, T. P. Russell and C. J. Hawker, Adv. Mater., 2003, 15, 297-300.

27 K. R. Thomas, A. Ionescu, J. Gwyther, I. Manners, C. H. W. Barnes, U. Steiner and E. Sivaniah, J. Appl. Phys., 2011, 109, 073904.

28 M. Mazurowski, M. Gallei, J. Li, H. Didzoleit, B. Stühn and M. Rehahn, Macromolecules, 2012, 45, 8970-8981.

29 D. Scheid, G. Cherkashinin, E. Ionescu and M. Gallei, Langmuir, 2014, 30, 1204-1209.

30 J. Elbert, H. Didzoleit, C. Fasel, E. Ionescu, R. Riedel, B. Stuhn and M. Gallei, Macromol. Rapid Commun., 2015, 36, 597-603.

31 S. Kaur, M. Gallei and E. Ionescu, Adv. Polym. Sci., 2015, 267, 143-185.

32 I. W. Hamley, The Physics of Block Copolymers, Oxford University Press, Oxford, 1998.

33 M. Lazzari, G. Liu and S. Lecommandoux, Block Copolymers in Nanoscience, Wiley-VCH, Weinheim, 2006.

34 N. A. Lynd, A. J. Meuler and M. A. Hillmyer, Prog. Polym. Sci., 2008, 33, 875-893.

35 H.-C. Kim, S.-M. Park and W. D. Hinsberg, Chem. Rev., 2010, 110, 146-177.

36 D. Klinger, M. J. Robb, J. M. Spruell, N. A. Lynd, C. J. Hawker and L. A. Connal, Polym. Chem., 2013, 4, 5038-5042.

37 R. C. Hayward and D. J. Pochan, Macromolecules, 2010, 43, 3577-3584.

38 F. H. Schacher, P. A. Rupar and I. Manners, Angew. Chem., Int. Ed., 2012, 51, 7898-7921.

39 J. Zhou, G. R. Whittell and I. Manners, Macromolecules, 2014, 47, 3529-3543.

40 C. G. Hardy, L. Ren, S. Ma and C. Tang, Chem. Commun., 2013, 49, 4373-4375.

41 J. Zhang, J. Yan, P. Pageni, Y. Yan, A. Wirth, Y. P. Chen, Y. Qiao, Q. Wang, A. W. Decho and C. Tang, Sci. Rep., 2015, 5, 11914.

42 P. Chadha and P. J. Ragogna, Chem. Commun., 2011, 47, 5301-5303.

43 M. Hadadpour, Y. Liu, P. Chadha and P. J. Ragogna, Macromolecules, 2014, 47, 6207-6217.

44 R. Gleiter and G. Pflästerer, Organometallics, 1993, 12, 1886-1889.

45 M. S. Sigman, A. W. Fatland and B. E. Eaton, J. Am. Chem. Soc., 1998, 120.

46 C. E. Anderson, L. E. Overman, C. J. Richards, M. P. Watson and N. White, Org. Synth., 2007, 84, 139-147.

47 A. Faldi, M. Tirrell, T. P. Lodge and E. v. Meerwall, Macromolecules, 1994, 27, 4184-4192.
48 M. Gallei, R. Klein and M. Rehahn, Macromolecules, 2010, 43, 1844-1854.

49 R. W. G. Wyckoff, Crystal Structures, Interscience Publishers, New York, 2nd edn, 1963.

50 V. G. Hadjiev, M. N. Iliev and I. V. Vergilov, J. Phys. C: Solid State Phys., 1988, 21, L199-L201.

51 D. Gallant, M. Pezolet and S. Simard, J. Phys. Chem. B, 2006, 110, 6871-6880.

52 C. K. Xu, Y. K. Liu, G. D. Xu and G. H. Wang, Chem. Phys. Lett., 2002, 366, 567-571.

53 C. A. Melendres and S. Xu, J. Electrochem. Soc., 1984, 131, 2239-2243.

54 H. C. Choi, Y. M. Jung, I. Noda and S. B. Kim, J. Phys. Chem. B, 2003, 107, 5806-5811.

55 J. Llorca, P. R. de la Piscina, J. A. Dalmon and N. Homs, Chem. Mater., 2004, 16, 3573-3578.

56 C. W. Tang, C. B. Wang and S. H. Chien, Thermochim. Acta, 2008, 473, 68-73.

57 J. S. Gwag and Y. Sohn, Bull. Korean Chem. Soc., 2012, 33, 505-510.

58 H. Luo, D. Wang, J. He and Y. Lu, J. Phys. Chem. B, 2005, 109, 1919-1922.

59 P. Poizot, S. Laruelle, S. Grugeon, L. Dupont and J.-M. Tarascon, Nature, 2000, 407, 496-499.

60 Y. Ma, W.-F. Dong, M. A. Hempenius, H. Möhwald and G. J. Vancso, Nat. Mater., 2006, 5, 724-729.

61 X. Feng, H. Wu, X. Sui, M. A. Hempenius and G. Julius Vancso, Eur. Polym. J., 2015, 72, 535-542.

62 L. Ren, C. G. Hardy, S. Tang, D. B. Doxie, N. Hamidi and C. Tang, Macromolecules, 2010, 43, 9304-9310.

63 K. Zhang, X. Feng, X. Sui, M. A. Hempenius and G. J. Vancso, Angew. Chem., Int. Ed. Engl., 2014, 53, 1378913793.

64 R. H. Staff, M. Gallei, K. Landfester and D. Crespy, Macromolecules, 2014, 47, 4876-4883.

65 T. Brotin, L. Pospíšil, J. Fiedler, B. T. King and J. Michl, J. Phys. Chem. B, 1998, 102, 10062-10070.

66 U. Koelle, Inorg. Chim. Acta, 1981, 47, 13-18.

67 N. McGrath, F. H. Schacher, H. Qiu, S. Mann, M. A. Winnik and I. Manners, Polym. Chem., 2014, 5, 1923-1929.

68 Z. M. Hudson, C. E. Boott, M. E. Robinson, P. A. Rupar, M. A. Winnik and I. Manners, Nat. Chem., 2014, 6, 893898.

69 C. Tonhauser, M. Mazurowski, M. Rehahn, M. Gallei and H. Frey, Macromolecules, 2012, 45, 3409-3418.

70 J. Morsbach, A. Natalello, J. Elbert, S. Winzen, A. Kroeger, H. Frey and M. Gallei, Organometallics, 2013, 32, 60336039. 